\title{
The three-dimensional Cauchy-condition surface method to reconstruct the last closed magnetic surface in non-axisymmetric fusion plasma
}

\author{
M. Itagaki ${ }^{1} \&$ K. Watanabe ${ }^{2}$ \\ ${ }^{I}$ Faculty of Engineering, Hokkaido University, Japan \\ ${ }^{2}$ National Institute for Fusion Science, Japan
}

\begin{abstract}
The three-dimensional (3D) Cauchy-condition surface (CCS) method code is now under development to reconstruct the $3 \mathrm{D}$ magnetic field profile outside a non-axisymmetric fusion plasma using only magnetic sensor signals. The boundary integral equations in terms of $3 \mathrm{D}$ vector potential for magnetic field sensors, flux loops and points along the CCS are solved simultaneously. Test calculations have been carried out for the Large Helical Device (LHD). The magnetic field line tracing based on the reconstructed field indicates the plasma boundary (the outer surface of the stochastic region) precisely. The Poincare plot points obtained from a field line tracing are converted to contours of a 'quasi' magnetic surface function using the expansion of radial basis functions. Introducing the 'inside/outside' ratio related to the scatter in the Poincare plot, the contour where the ratio jumps is taken as a best estimate for the location of the last closed magnetic surface (LCMS). This reconstructed LCMS agrees well with the reference LCMS.

Keywords: magnetic sensor, plasma boundary, last closed magnetic surface, Cauchy-condition surface method, twisted Cauchy-condition surface, magnetic field line tracing, Poincaré plot.
\end{abstract}

\section{Introduction}

In a nuclear fusion device, the plasma boundary shape is one of the important parameters to identify the MHD equilibrium configuration. The Cauchy- 
condition surface (CCS) method [1,2] is a computer-aided approach to identify the plasma boundary shape only using signals of magnetic sensors located outside the plasma. The method has been established for operating control and diagnosis of tokamak-type devices. However, it has never been applied for the non-axisymmetric plasma in a helical type device such as the Large Helical Device (LHD), Japan.

For such non-axisymmetric plasmas, 3Dimensional (3D) analyses are required. Extension of the CCS method to a $3 \mathrm{D}$ space is quite challenging. It requires a huge number of unknowns, so that it needs a large number of sensors, and the problem becomes ill-conditioned. Next, for a tokamak the magnetic surface function $\psi$ that satisfies $\mathbf{B} \cdot \nabla \psi=0$ has a simple form of $\psi=r A_{\varphi}$ with the toroidal component of the vector potential $A_{\varphi}$; however, there is no mathematical expression of the surface function for a helical-type device. Because of this, we need to perform a magnetic field line tracing based on the reconstructed magnetic field to identify the shape of the plasma boundary [3, 4].

The last closed magnetic surface (LCMS) in the present title means the outermost closed surface that is recognized through a magnetic field line tracing. Even outside the LCMS there exist field lines that reach the divertor plate. This field line region is called the 'stochastic region'. The CCS method solutions are valid only for the fully open field line region. Even in the stochastic region currents exist, but they are so weak that the solution accuracy is fair. The 'dirty' region is now defined as the domain sandwiched between the LCMS and the CCS. As the strong current exists in this region, the solution has a large error.

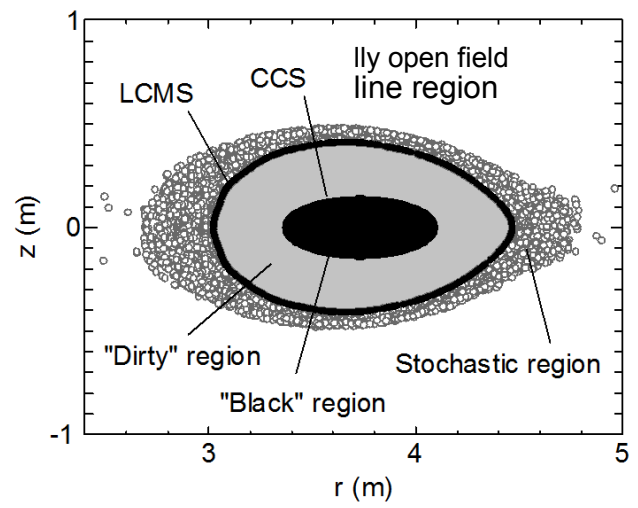

Figure 1: Definitions of the four regions.

\section{Outline of the 3D CCS method}

The Cauchy-condition surface (CCS), where both the Dirichlet and the Neumann conditions are unknown, is hypothetically placed in a domain that can be supposed to be inside the plasma. In the analysis, no plasma current is assumed outside this CCS, where in reality plasma current does exist. Instead, the CCS plays the same role as the plasma current in causing the field outside the plasma. 
Due to the vacuum field assumption, the solution near the CCS is dirty, and this exerts a harmful influence on the solution even outside the LCMS if the cross section of the CCS is large. To avoid this difficulty, we have introduced the 'twisted CCS' [4] shown in fig. 2. In this model, an elliptic cross section rotates with the variation in vacuum vessel geometry in the toroidal direction. Independent of the toroidal angle, the twisted CCS can keep a certain distance from its surface to the LCMS. A reduction in the numerical error can then be expected.

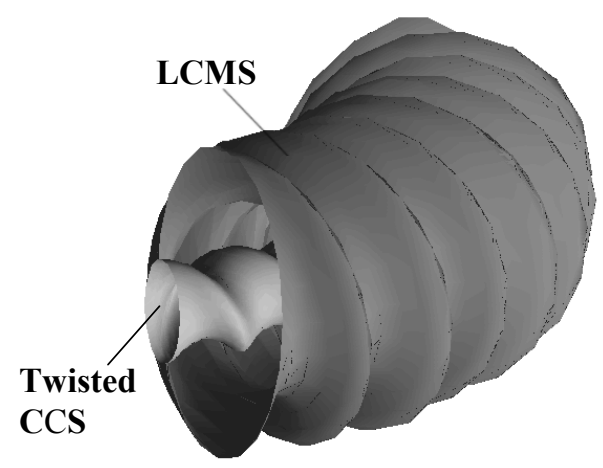

Figure 2: Image of the twisted CCS.

A 3D Cartesian coordinate system is adopted to realize a boundary-only integral formulation [3]. The first step of the analysis is to solve the following boundary integral equations (BIEs) and obtain the values of the vector potential and its derivative on the CCS in such a way that they will be consistent with the sensor signals $[3,4]$.

(i) For magnetic field sensor locations $i$ :

$$
\begin{aligned}
B_{j}-W_{j}^{(B)}=\sum_{k} \int_{\Gamma_{\mathrm{CCS}}}\left\{\left(L_{k}^{j} \phi_{i}^{*}\right) \frac{\partial A_{k}}{\partial n}-A_{k}\left(L_{k}^{j} \frac{\partial \phi_{i}^{*}}{\partial n}\right)\right\} d \Gamma . \\
\quad(j=r, \varphi, z ; k=x, y, z)
\end{aligned}
$$

The operator $L_{k}^{j}$ corresponds to a component in $\mathbf{B}=\nabla \times \mathbf{A}$ with $\mathbf{A}$ expressed in Cartesian coordinates, $W_{j}^{(B)}$ is the contribution of external coil currents, and $\phi_{i}^{*}$ the fundamental solution of the Laplace equation.

(ii) For flux loops:

e.g., the BIE for a circle loop set in the toroidal direction is given as

$$
\psi^{(\text {Tor })}-W^{(\text {Tor })}=\sum_{k} \int_{\Gamma_{\mathrm{cCS}}}\left\{\frac{\partial A_{k}}{\partial n}\left(\int_{0}^{2 \pi} \eta_{k} \phi_{i}^{*} d \varphi\right)-A_{k}\left(\int_{0}^{2 \pi} \eta_{k} \frac{\partial \phi_{i}^{*}}{\partial n} d \varphi\right)\right\} d \Gamma
$$


with $\eta_{x}=-R \sin \varphi$ and $\eta_{y}=R \cos \varphi$ for the radius $R$ of the circle loop.

(iii) For points $i$ on the $\operatorname{CCS}\left(\Gamma_{C C S}\right)$ :

$$
\frac{1}{2} A_{k, i}=\int_{\Gamma_{C C S}}\left(\phi_{i}^{*} \frac{\partial A_{k}}{\partial n}-A_{k} \frac{\partial \phi_{i}^{*}}{\partial n}\right) d \Gamma . \quad(k=x, y, z)
$$

The above three types of BIEs are discretized, coupled and converted to a matrix equation that has the form

$$
\mathbf{D p}=\mathbf{g},
$$

where the solution vector $\mathbf{p}$ contains the vector potentials $\mathbf{A}$ and their normal derivatives $\partial \mathbf{A} / \partial n$ on the CCS. This equation is solved using the singular value decomposition (SVD) technique [5]. The Truncated SVD technique was employed to obtain a regularized solution.

Considering the 10 -fold rotational symmetry of the LHD in the toroidal direction, the number of unknowns is reduced by a factor of 10 with the aid of a linear transformation of the vector potential expressed in the Cartesian coordinate system $[3,4,6]$.

Once all the values of these conditions on the CCS are known, the magnetic fields for arbitrary points outside the CCS can be calculated using the formula

$$
\begin{aligned}
B_{j}=\sum_{k} \int_{\Gamma_{\mathrm{CCS}}}\left\{\left(L_{k}^{j} \phi_{i}^{*}\right) \frac{\partial A_{k}}{\partial n}-A_{k}\left(L_{k}^{j} \frac{\partial \phi_{i}^{*}}{\partial n}\right)\right\} d \Gamma+W_{j}^{(B)} . \\
(j=r, \varphi, z ; \quad k=x, y, z)
\end{aligned}
$$

After the 3D magnetic field distribution has been obtained, the magnetic field line can be traced. A magnetic line of force satisfies the equations

$$
\frac{d r}{B_{r}}=\frac{r d \varphi}{B_{\varphi}}=\frac{d z}{B_{z}}=\frac{d l}{B},
$$

where $l$ is the length along a magnetic line of force, i.e.,

$$
(d l)^{2}=(d r)^{2}+(r d \varphi)^{2}+(d z)^{2} .
$$

In the present research, this trace is performed using the MGTRC code [7]. Magnetic fields at any points are interpolated using a 3D 4th order spline function. Equations (6) are integrated with the use of an 8-stage 6th order RungeKutta formulation.

\section{Numerical demonstration}

One considers the plasma with the volume-averaged $\beta$ being $\langle\beta\rangle=2.7 \%$ in the LHD. The reference MHD equilibrium had been analyzed beforehand using the 3D MHD equilibrium calculation code HINT2 [8]. That is, the magnetic sensor signals and the magnetic field caused by the external coil currents are known before the present analysis. The reconstructed results are compared with the reference solutions. 


\subsection{Calculation conditions}

Magnetic sensors are arranged a little way outside the LCMS as shown in fig. 3 . We assumed 126 flux loops and 440 field sensors. Each of the field sensors is assumed to detect all of the 3 components of magnetic field. The CCS was set in the domain that can be supposed to be inside the plasma. Considering a 10-fold rotational symmetry in the toroidal direction, only a 36-deg. portion of the CCS torus was modeled and this portion was divided into 48 boundary elements, each of which has 9 nodal points.

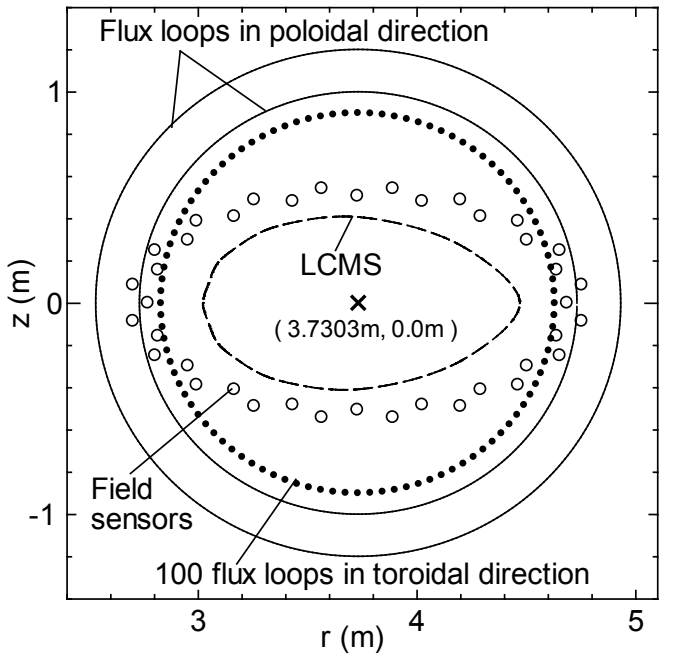

Figure 3: Sensor locations on the horizontally elongated cross-section.

\subsection{Reconstructed magnetic field profile}

Figure 4 shows the profile of absolute error of the reconstructed $B_{\varphi}$ (the toroidal component of the field) on the horizontally elongated cross section. The

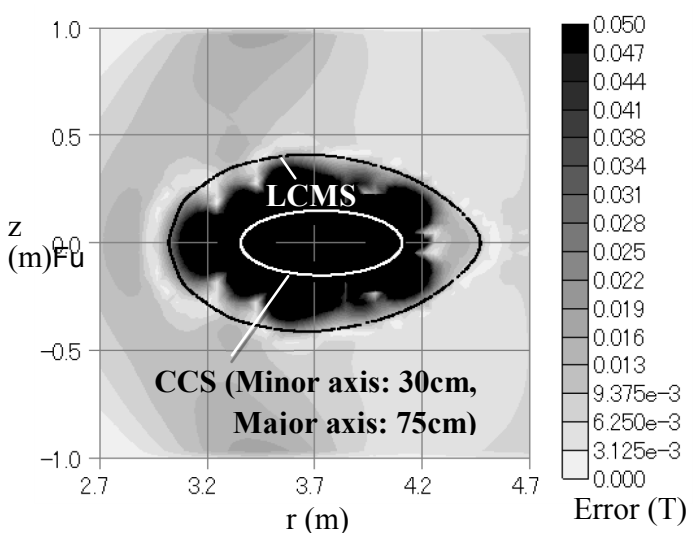

Figure 4: Distribution of absolute error of $B_{\varphi}$. 
reference solutions were provided beforehand using the HINT2 code. The errors larger than $0.05 \mathrm{~T}$ are limited inside the LCMS, i.e., inside the 'dirty' region.

\subsection{Magnetic field line tracing}

The magnetic field line tracing started at the points $(r, z, \varphi)$ :

$$
r=4.30+0.01 k \text { in }[\mathrm{m}] \text { with } k=0,1, \cdots, 40,
$$

$z=0.0 \mathrm{~m}$ and $\varphi=18^{\circ}$ (the horizontally elongated cross section). Figure 5(a) shows the Poincaré plots of the field lines on the horizontally elongated cross section.

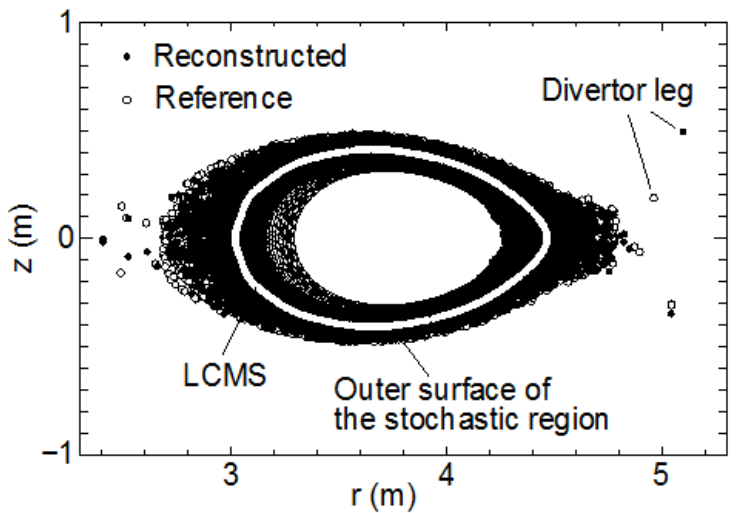

(a) Stochastic region

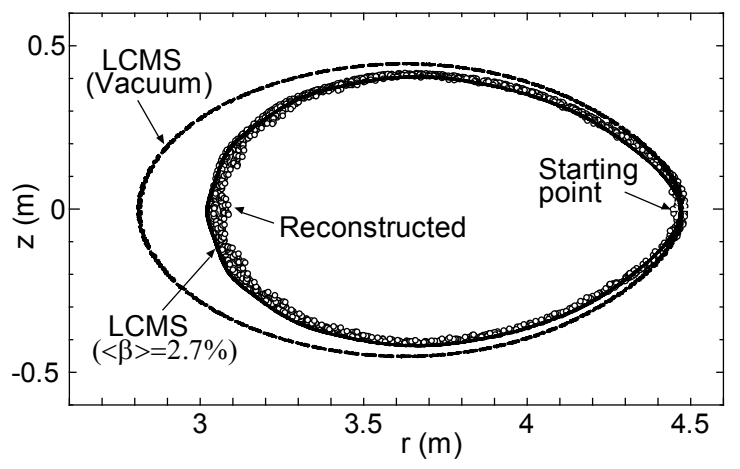

(b) LCMS

Figure 5: $\quad$ Poincaré plots of the field line.

The reconstructed plasma boundary (the outer surface of the stochastic region) shows a good agreement with the reference one. This is supported by the mathematical background that outside the plasma the CCS method solution agrees with the solution based on the existence of plasma current (see the Appendix in Ref. [3]). 
Figure 5(b) highlights the Poincaré plot originating at the starting point, $r=4.47 \mathrm{~m}$, which formed the LCMS when following the reference field. The dashed line shows the LCMS for the vacuum field. This is shifted outward when $\langle\beta\rangle$ takes the nonzero value, $2.7 \%$. The reconstructed plots are distributed along the reference LCMS for $\langle\beta\rangle=2.7 \%$ (the solid line). However, they do not form a sharp closed surface.

\subsection{Numerical determination of the LCMS}

The Poincaré plot based on the reconstructed field did not form the LCMS clearly. In this section we discuss an idea to estimate where the LCMS is. With the use of the radial basis function (RBF) expansion

$$
\psi(r, z)=\sum_{i=1}^{N} w_{i} f_{i}\left(r, z ; r_{i}, z_{i}\right)
$$

based on the Gaussian type RBF

$$
f_{i}\left(r, z ; r_{i}, z_{i}\right)=\exp \left\{-\left(\left(r-r_{i}\right)^{2}+\left(z-z_{i}\right)^{2}\right) / \sigma^{2}\right\},
$$

the Poincaré plot is converted into a contour map of quasi-magnetic surfaces that correspond to the starting points in the field line tracing. The starting points are located on a straight line (see eqn (7)), so that the r-coordinate of starting points can be recognized as a quasi-magnetic surface function. In fig. 6 the closed surfaces are found even outside the LCMS, but they are not the true magnetic surfaces. We need to dismiss them to determine the true LCMS.

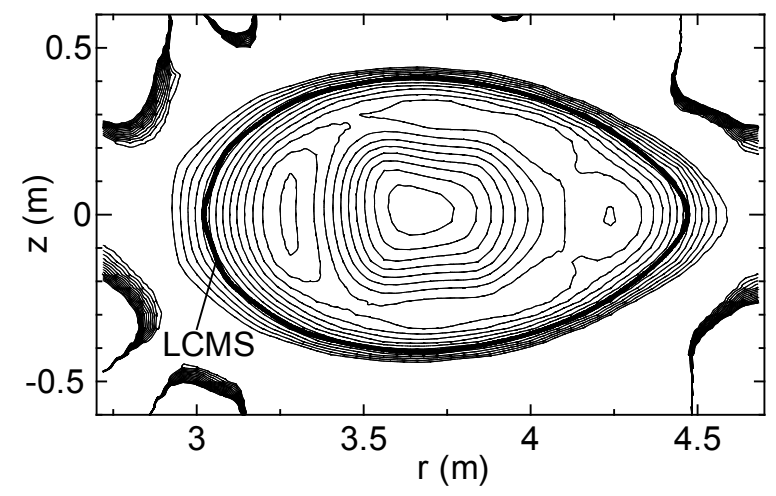

Figure 6: Contours of RBF-built function.

Figures 7(a) and 7(b) show the Poincaré plots originating at two starting points. For the r-coordinates of starting point, $4.47 \mathrm{~m}$ (see fig. $7(\mathrm{a})$ ), the plots (black dots) are slightly scattered along the corresponding quasi-magnetic surface (the white closed line). However, in case of $4.46 \mathrm{~m}$ (see fig. 7(b)) where 
the quasi-magnetic surface (in white) is inside the LCMS, i.e., the dirty region, the scatter inside the quasi-magnetic surface is much larger than outside the surface. This difference can be used as an indicator to judge where the LCMS is.

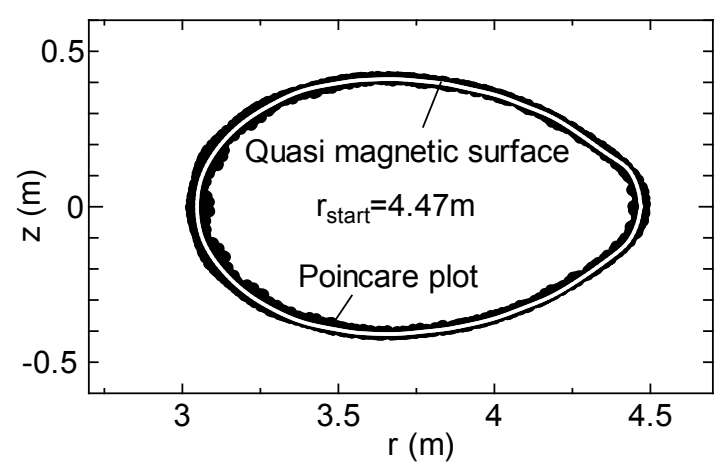

(a) $r_{\text {start }}=4.47 \mathrm{~m}$

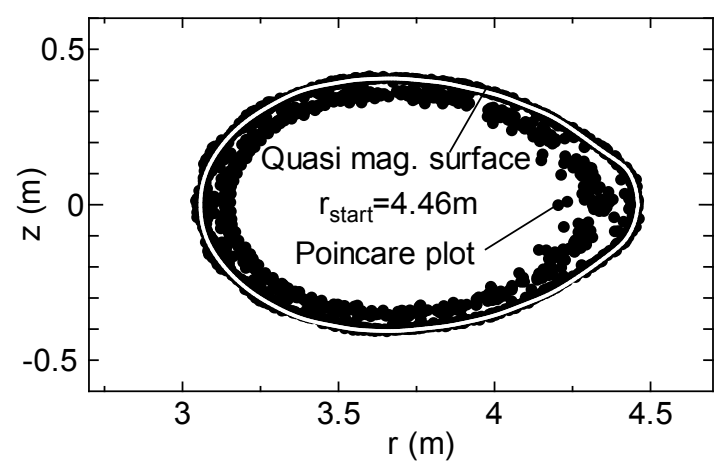

(b) $r_{\text {start }}=4.46 \mathrm{~m}$

Figure 7: Poincaré plot with the quasi magnetic surface near the LCMS.

We here introduce the 'scatter' given by

$$
s^{2}=\frac{1}{m} \sum_{j=1}^{m} d_{j}^{2},
$$

where $d_{j}$ denotes the distance between the reconstructed magnetic surface and the $j$-th point in the $m$ Poincaré plot points.

We define this 'inside/outside' ratio with the scatters for points inside and the outside the contour under consideration. Figure 8 shows the variation in this ratio as a function of r-coordinate of the starting point. The ratio jumps at $r=4.47 \mathrm{~m}$, which corresponds to the LCMS, the entrance to the 'dirty region'. 
The contour corresponding to $r=4.47 \mathrm{~m}$ was extracted from the contours of quasi-magnetic surfaces drawn by the RBF expansion. As shown in fig. 9, the extracted (reconstructed) LCMS agrees well with the reference LCMS.

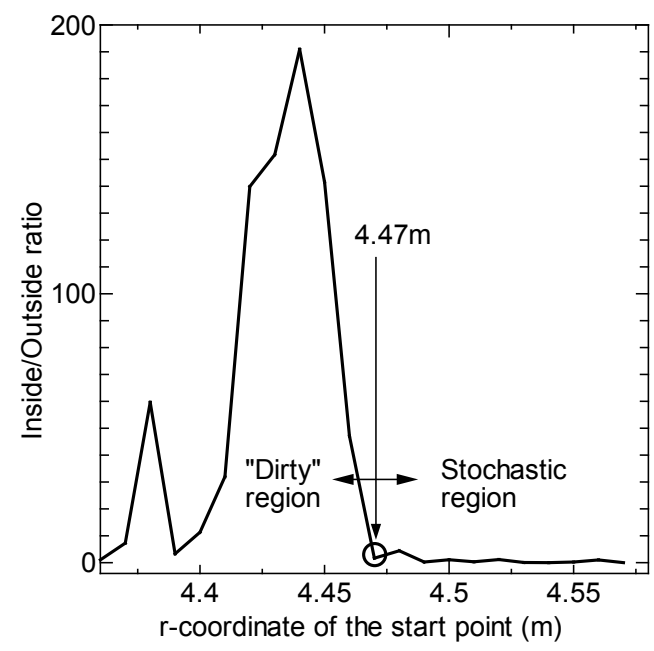

Figure 8: Variation in the 'inside/outside' ratio.

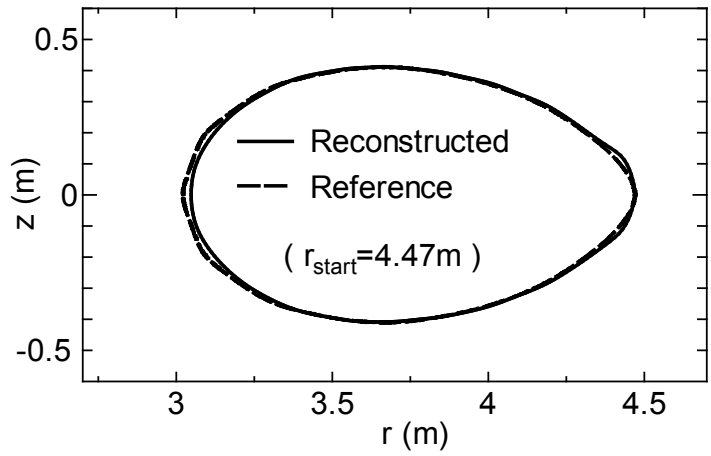

Figure 9: Reconstructed LCMS.

\section{Conclusion}

A prototype of 3D CCS method code has been developed. With the use of the 'twisted CCS', the field distribution outside the non-axisymmetric plasma can be reconstructed with a fairly acceptable accuracy. The field line trace indicates the outer surface of stochastic region precisely. The LCMS can be identified using the numerical process proposed here. It should be stressed that they were reconstructed using only sensor signals. 
The authors are now considering the following as further tasks:

- Reduction of the number of unknowns and hence the number of the sensor signals

- More numerical demonstrations to validate the proposed idea of numerical determination of LCMS

- Guidelines for the best regularization in the SVD technique are still worth investigating.

\section{Acknowledgements}

This research was performed with the support and under the auspices of the NIFS Collaboration Research Program (NIFS08KLPP015). This work was also supported by the Ministry of Education, Culture, Sports, Science and Technology, Grant-in-Aid for Scientific Research (C), 24561019, 2012.

\section{References}

[1] Kurihara, K. 2000 Fusion Eng. Des. 51-52 1049

[2] Itagaki, M., Yamaguchi, S. and Fukunaga, T. 2005 Nucl. Fusion 45153

[3] Itagaki, M., Maeda, T., Ishimaru, T., Okubo, G., Watanabe, K., Seki, R. and Suzuki, Y. 2011 Plasma Phys. Control. Fusion 53105007

[4] Itagaki, M., G. Okubo, G., Akazawa, M., Matsumoto, Y., Watanabe, K., Seki, R. and Suzuki, Y., 2012 Plasma Phys. Control. Fusion 54125003

[5] Press, W.H., Flannery, B.P., Teukolsky, S.A. and Vetterling, W.T. 1986 Numerical Recipes - The Art of Scientific Computing (Cambridge: Cambridge University Press)

[6] Itagaki, M., Ishimaru, T. and Watanabe, K. 2010 Proc. the 32nd International Conf. on Boundary Elements and Other Mesh Reduction Methods, (Southampton, U.K., 2010), (WIT Press, Southampton, U.K.) p.133

[7] LHD Experimental Board 2009 Section 6 LHD Experiment Technical Guide 2009 (Toki, Japan: National Institute for Fusion Science)

[8] Suzuki, Y., Nakajima, N., Watanabe, K., Nakamura, Y. and Hayashi, T. 2006 Nucl. Fusion 46 L19 